\title{
Consecuencias hemodinámicas y respiratorias del síndrome compartimental abdominal en un modelo experimental
}

\author{
FRANCO DÍAZ R. ${ }^{1}$, ALEJANDRO DONOSO F. ${ }^{1}$, CRISTÓBAL CARVAJAL B. ${ }^{1}$, \\ TATIANA SALOMÓN S. ${ }^{2}$, MARÍA FERNANDA TORRES G. ${ }^{2}$, \\ BENJAMÍN ERRANZ M. ${ }^{3}$, PABLO CRUCES R. ${ }^{1}$ \\ 1. Pediatra, Medicina Intensiva Infantil. \\ 2. Enfermería Intensiva Infantil. \\ 3. Licenciado en Ingeniería en Biotecnología Molecular. \\ Departamento de Pediatría Facultad de Medicina Clínica Alemana-Universidad del Desarrollo. Hospital Padre Hurtado.
}

\begin{abstract}
Hemodynamic and respiratory alterations in an experimental abdominal compartment syndrome model

Introduction: Abdominal compartment syndrome (ACS) is a severe and under-reported condition among the pediatric population due to inadequate warning and recognition. It can be caused by medical and surgical reasons, resulting in a high mortality rate. Objective: To determine the magnitude of the initial hemodynamic and respiratory consequences triggered by the induction of ACS in an experimental model. Methods: The model consisted of twelve anesthetized pigs $(4.8 \pm 0.1 \mathrm{~kg})$. The ACS was induced by instillation of colloid solution in the peritoneal cavity to obtain an intra-abdominal pressure (IAP) of $24.9 \pm 0.6 \mathrm{mmHg}$. In basal conditions and after the ACS induction, a conventional hemodynamic monitoring and transpulmonary thermodilution were performed. At the same time, arterial blood gases and lung mechanics analysis were measured. Results: There was a reduction of cardiac output by $16 \%\left(5.19 \pm 0.33\right.$ to $\left.4.34 \pm 0.28 \mathrm{l} / \mathrm{min} / \mathrm{m}^{2}, \mathrm{p}=0.01\right)$ and abdominal perfusion pressure by $20 \%(72.3 \pm 3.2$ to $57.3 \pm 4.0 \mathrm{mmHg}, \mathrm{p}<0.001)$ without changes in heart rate, arterial or central venous pressure. In addition there was an approximately $50 \%$ worsening of respiratory system compliance $\left(1.28 \pm 0.09\right.$ to $\left.0.62 \pm 0.04 \mathrm{ml} / \mathrm{cmH}_{2} \mathrm{O} / \mathrm{kg}, \mathrm{p}=0.002\right)$ associated with a significant increase in intrathoracic pressure and slight decrease in oxygenation. Discussion: In this experimental model, the early development of hemodynamic and pulmonary dysfunction could be observed. A reduction of cardiac output that was not detected by conventional monitoring and a substantial deterioration of lung mechanics, characteristic of restrictive disease, associated with mild alterations in gas exchange were reported. It is essential then to monitor the IAP in patients predisposed to develop ACS, especially in the case of organ dysfunction deterioration, as severe hypotension and hypoxemia are late signs of this complication.
\end{abstract}

Recibido el 27 de febrero de 2012, devuelto para corregir el 29 de junio de 2012, segunda versión el 08 de julio de 2012 , aceptado para publicación el 12 de agosto de 2012.

Estudio financiado por proyecto SOCHIPE 2008009.

Este trabajo cumple con los requisitos sobre consentimiento /asentimiento informado, comité de ética, financiamiento, estudios animales y sobre la ausencia de conflictos de intereses según corresponda.

Correspondencia a:

Dr. Pablo Cruces R.

E-mail: pcrucesr@gmail.com 
(Key words: Abdominal compartment syndrome, intra-abdominal pressure, abdominal perfusion pressure, hemodynamics, lung function).

Rev Chil Pediatr 2012; 83 (5): 454-461

\section{RESUMEN}

Introducción: El síndrome compartimental abdominal (SCA) es una entidad grave, de escaso reporte en población pediátrica por una inadecuada alerta y reconocimiento. Puede ser originado por causas médicas y quirúrgicas, presentando una elevada mortalidad. Objetivo: Determinar la magnitud de las consecuencias hemodinámicas y respiratorias iniciales desencadenadas por la inducción de un SCA en un modelo experimental. Método: Doce cerdos anestesiados $(4,8 \pm 0,1 \mathrm{~kg})$. El SCA fue inducido con instilación de solución coloide en cavidad peritoneal para obtener una presión intra-abdominal (PIA) de $25 \pm 5 \mathrm{mmHg}$. En condiciones basales y posterior a inducción del SCA se realizó monitorización hemodinámica convencional y termodilución transpulmonar. Paralelamente se midió gasometría arterial y análisis de mecánica pulmonar. Resultados: Hubo una reducción del gasto cardíaco en $16 \%\left(5,19 \pm 0,33\right.$ a 4,34 $\left.\pm 0,281 / \mathrm{min} / \mathrm{m}^{2}, \mathrm{p}=0,01\right)$ y de la presión de perfusión abdominal en $20 \%(72,3 \pm 3,2$ a $57,3 \pm 4,0 \mathrm{mmHg}, \mathrm{p}<0,001)$ sin cambios en frecuencia cardiaca, presión arterial y venosa central. Además ocurrió un deterioro de la compliance del sistema respiratorio cercana al $50 \%$ $\left(1,28 \pm 0,09\right.$ a $\left.0,62 \pm 0,04 \mathrm{ml} / \mathrm{cmH}_{2} \mathrm{O} / \mathrm{kg}, \mathrm{p}=0,002\right)$ asociado a un incremento significativo en las presiones intratorácicas y disminución leve de la oxigenación. Discusión: En este modelo experimental se pudo apreciar el desarrollo temprano de disfunción hemodinámica y pulmonar. Se evidenció una reducción de gasto cardiaco no detectado por la monitorización convencional y un deterioro substancial de la mecánica pulmonar, propia de una enfermedad restrictiva, asociado a alteraciones leves del intercambio gaseoso. Creemos que es fundamental monitorizar la PIA en pacientes predispuestos a desarrollar un SCA, más aún ante empeoramiento de disfunciones orgánicas dado que la hipotensión e hipoxemia grave son signos tardíos de esta complicación.

(Palabras clave: Síndrome compartimental abdominal, presión intra-abdominal, presión de perfusión abdominal, hemodinamia, función pulmonar).

Rev Chil Pediatr 2012; 83 (5): 454-461

\section{Introducción}

El síndrome compartimental del abdomen (SCA) es el resultado final de un proceso iniciado con el aumento persistente de la presión intra-abdominal (PIA) en una magnitud tal que es capaz de alterar el influjo vascular regional, pudiendo culminar en falla orgánica múltiple (FOM) y una mortalidad entre 40 y $60 \%$ en población pediátrica ${ }^{1-3}$. Desde el punto de vista clínico, SCA se define como un PIA por encima de $20 \mathrm{mmHg}$ con evidencia de disfunción/ fracaso de órganos ${ }^{4}$. A pesar que la incidencia reportada de SCA es relativamente baja en niños críticamente enfermos $(0,6-4,7 \%)$, este síndrome pareciera ser escasamente comunicado por inadecuada alerta y reconocimiento ${ }^{5-7}$.

En un inicio se pensó que el SCA afectaba primariamente a pacientes con patologías abdominales traumáticas y quirúrgicas, no obstante estudios recientes han identificado esta complicación en niños y adultos con patología crítica médica $^{1-10}$. No existe un valor absoluto de PIA que defina el momento exacto en que se desarrolla un SCA, por lo que la acuciosidad de las mediciones, la evolución temporal y el desarrollo de nuevas disfunciones de órganos, son de importancia vital en la toma de decisiones terapéuticas ${ }^{11}$.

Basado en diversos trabajos experimentales, se ha determinado que un incremento grave de la PIA induce un deterioro de la función pulmonar, cardiovascular, reducción de la presión de perfusión renal y cerebral, entre otros, hallazgos corroborados en adultos críticamente enfermos ${ }^{12-14}$. La magnitud de las disfunciones orgánicas desencadenadas por el desarrollo súbito de un SCA no ha sido evaluada en profundidad en pediatría. El estudio de esta entidad en modelos pediátricos es fundamental, dadas las diferencias relevantes en la fisiología respiratoria y cardiovascular entre niños y adultos ${ }^{15,16}$.

Nuestro objetivo es determinar la magnitud 
de las consecuencias hemodinámicas y respiratorias iniciales desencadenadas por la inducción de un síndrome compartimental abdominal en un modelo experimental pediátrico.

\section{Material y Métodos}

El protocolo experimental fue aprobado por el Comité de Ética Facultad de Medicina Clínica Alemana-Universidad del Desarrollo. Los animales recibieron cuidados en conformidad con los "Guiding Principles in the Care and Use of Laboratory Animals" adoptados por la American Physiological Society.

El estudio se realizó en doce cerdos Large-White, menores de un mes de vida, de 4,8 $\pm 0,15 \mathrm{~kg}$, que fueron premedicados con ketamina $20 \mathrm{mg} / \mathrm{kg}$ y acepromazina $1,1 \mathrm{mg} / \mathrm{kg}$ intramuscular para los procedimientos iniciales. Durante el resto del protocolo experimental la anestesia fue mantenida con una infusión continua de propofol $(10 \mathrm{mg} / \mathrm{kg} / \mathrm{h})$ y fentanyl $(4 \mu \mathrm{g} /$ $\mathrm{kg} / \mathrm{h})$, adicionándose pancuronium $(0,2 \mathrm{mg} /$ $\mathrm{kg} / \mathrm{h}$ ) como relajante neuromuscular. La hidratación fue mantenida con solución salina normal a $10 \mathrm{ml} / \mathrm{kg} / \mathrm{h}$. La temperatura se mantuvo a $37,0 \pm 0,7^{\circ} \mathrm{C}$ usando métodos convectivos convencionales.

En el protocolo de ventilación, se empleó ventilador mecánico EVITA XL (Dräger Me- dical, Germany) en modo controlado por volumen con los siguientes parámetros: volumen corriente $\left(\mathrm{V}_{\mathrm{T}}\right) 10 \mathrm{ml} / \mathrm{kg}$, PEEP de $5 \mathrm{~cm} \mathrm{H} \mathrm{H}_{2} \mathrm{O}$, frecuencia respiratoria (FR) 20 por min, tiempo inspiratorio (TI) $0,75 \mathrm{~s}$ y $\mathrm{FIO}_{2} 100 \%$.

Para la preparación quirúrgica, se infiltró en forma tópica lidocaína $1 \%(1 \mathrm{ml} / \mathrm{kg})$ y la tráquea fue canulada con un tubo de traqueostomía con cuff de 3,5 $\mathrm{mm}$ de diámetro interno (Mallinckrodt Shiley, St. Louis, MO). La vena yugular derecha fue denudada y se instaló un catéter (4F) (Arrow, Reading, PA, USA), procurando ubicación extra cardíaca en control radiológico. Con técnica similar se instaló catéter arterial (4F) con punta termistor en extremo distal (PiCCO ${ }^{\circledR}$ PV2014L08, Pulsion Medical Systems, Munich, Germany) en arteria axilar izquierda y se conectó a monitor $\mathrm{PiCCO}^{\circledR}$ plus para termodilución transpulmonar (TDTP). Finalmente se instaló catéter (7F) doble lumen (Arrow, Reading, PA, USA) en cavidad peritoneal (cuadrante inferior izquierdo), empleando técnica de Seldinger modificada. La PIA se midió a través de catéter peritoneal empleando la técnica descrita por Kron, a través de una interfase líquido-líquido ${ }^{17}$ (figura 1A).

Los signos vitales fueron monitorizados empleando el monitor Infinity Delta XL (Dräger Medical, Germany), registrando la frecuencia cardiaca (FC), presión arterial media (PAM), presión venosa central (PVC) y pre-

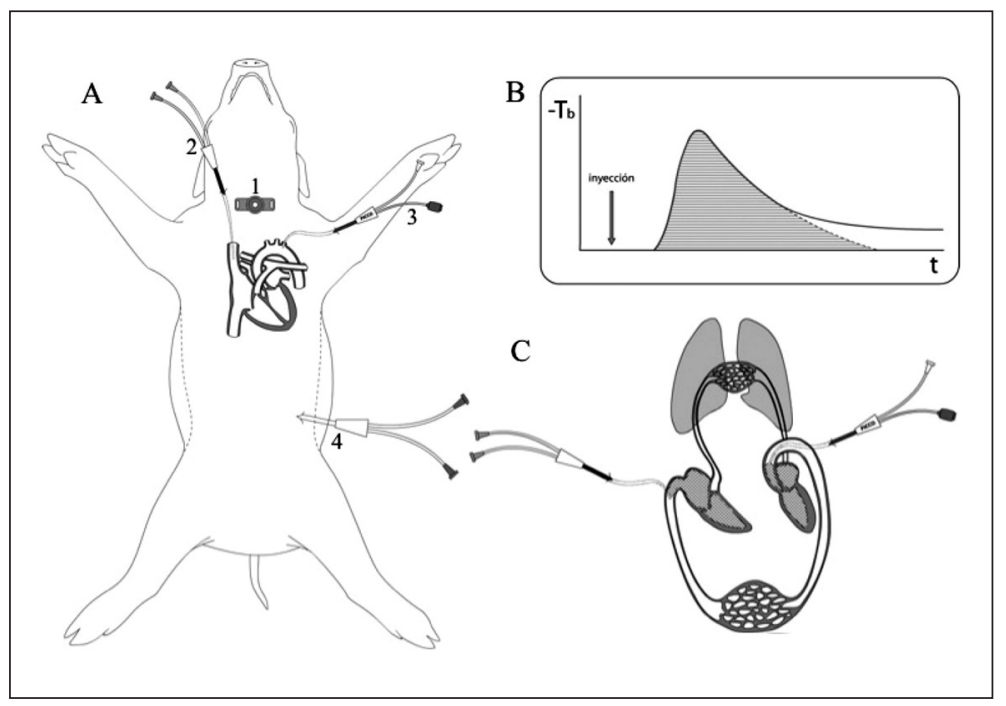

Figura 1. Monitorización de termodilución transpulmonar en el modelo experimental. A. Esquema de modelo experimental y su monitorización. 1. Cánula de traqueostomía. 2. Catéter Venoso Central en vena yugular interna derecha. 3. Catéter de termodilución $\mathrm{PiCCO}^{\circledR}$ en arteria axilar izquierda. 4. Catéter peritoneal. B. Curva de termodilución transpulmonar: el gasto cardiaco es calculado por el área bajo la curva, usando un algoritmo de Stewart-Hamilton modificado de la curva de termodilución. (T: temperatura; t: tiempo). C. Volumen Diastólico Final Global (GEDV) es el volumen de sangre contenido tetracameral del corazón. Es calculado mediante el análisis avanzado de la curva de termodilución. Es un indicador volumétrico de precarga. 
sión intra-abdominal. La presión de perfusión abdominal (PPA) se midió como la diferencia entre PAM y PIA.

Dado que está descrito que con niveles de PIA $>20 \mathrm{mmHg}$ existe un elevado riesgo de desarrollar daño tisular irreversible en pediatría ${ }^{5} \mathrm{y}$, en concordancia con una definición de consenso ${ }^{4}$, decidimos emplear un modelo experimental capaz de simular esta condición. Se realizó instilación de solución coloide isotónica (Voluven ${ }^{\circledR}$ 6\%, Fresenius-Kabi, Germany) en la cavidad peritoneal en alícuotas sucesivas de $100 \mathrm{ml}$, para obtener una PIA de $25 \pm 5$ $\mathrm{mmHg}$, a través del catéter previamente instalado.

Posterior a la instrumentación y previo al registro basal, la volemia fue corregida con bolos sucesivos de $10 \mathrm{ml} / \mathrm{kg}$ de solución coloide intravenosa, hasta no incrementar el GC más que un $10 \%$. Esta condición definió el estado de precarga óptima de cada sujeto. Se realizó un segundo registro tras mantener objetivo de presión intra-abdominal por un período de 120 min (figura 2).

Para la medición de gasto cardíaco, se realizó TDTP con $5 \mathrm{ml}$ de solución salina $0,9 \%$ fría $\left(<8^{\circ} \mathrm{C}\right)$ por catéter venoso yugular, administradas en forma aleatoria en el ciclo respiratorio. Las mediciones de gasto cardíaco (GC) fueron consideradas válidas cuando las tres medidas aisladas difirieron menos de un
$10 \%$ del promedio. En caso de una diferencia $>10 \%$, se realizó una medición adicional. Se consideraron valores indexados según superficie corporal. Área de superficie corporal fue calculada usando la siguiente fórmula: área de superficie corporal $=\mathrm{K} /$ peso (en kilogramos) $)^{2 / 3}$, donde $K=0,112$ para cerdos ${ }^{18}$. La entrega de oxígeno $\left(\mathrm{DO}_{2}\right)$ se calculó de acuerdo a la fórmula estándar. Simultáneamente se consignó precarga volumétrica (GEDV: del inglés global end diastolic volume, volumen diastólico final global), volumen sistólico indexado (VSI) y resistencia vascular sistémica indexada (IRVS) (figuras 1B y 1C).

Se midió gasometría arterial empleando cartridges i-STAT ${ }^{\circledR}$ EG6 (Abbott Laboratories, Princeton, $N J$ ) al inicio y luego de inducción del SCA. La presión diferencial fue calculada como la diferencia entre presiones inspiratoria (presión plateau, $\mathrm{P}_{\mathrm{PL}}$ ) y espiratoria final (PEEP total) de las vías aéreas, medidas tras una pausa inspiratoria y espiratoria 4 segundos respectivamente. La compliance estática del sistema respiratorio $\left(\mathrm{C}_{\mathrm{RS}}\right)$ fue calculada como la razón entre el $\mathrm{V}_{\mathrm{T}}$ espirado y la presión diferencial. La presión media de las vía aéreas (PMVA) fue registrada desde la pantalla del ventilador. El índice de oxigenación (IO) fue calculado como el producto entre la PMVA y la $\mathrm{FIO}_{2}$, dividido por la $\mathrm{PaO}_{2}$. Luego, aún bajo anestesia (Estado 3 - Plano 2), los animales

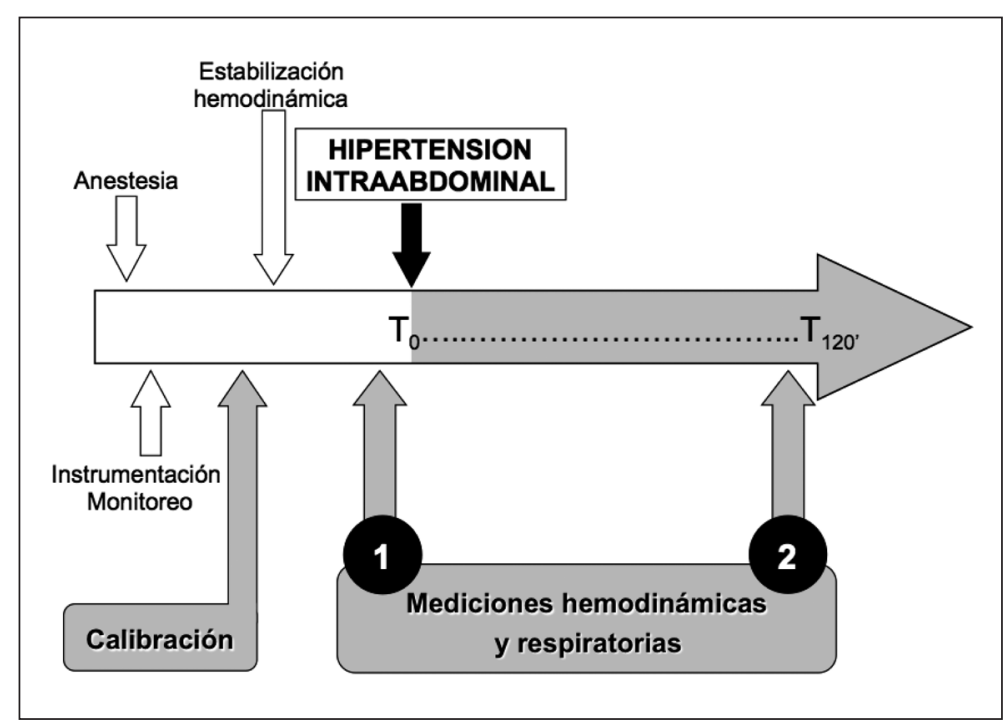

Figura 2. Diagrama experimental. 1: Mediciones basales, 2: Mediciones con hipertensión intra-abdominal. 
fueron eutanasiados por perfusión de cloruro de potasio al $10 \%$ hasta la detección de la fibrilación ventricular o asistolía.

Los valores fueron expresados como media \pm SEM. Se utilizó el test Shapiro-Wilk para evaluar la normalidad de las variables. Para comparar las variables estudiadas antes y después de la inducción de SCA se utilizó la prueba de Wilcoxon de rangos señalados y pares igualados para dos muestras dependientes. Se consideró significativo un $\mathrm{p}<0,05$. El análisis estadístico se realizó con programa SPSS $20^{\circledR}$ (SPPS, Chicago, Illinois, USA).

\section{Resultados}

Todos los animales completaron el protocolo experimental.

La PIA inicial fue 4,8 $\pm 0,4 \mathrm{mmHg}$. Se instiló en la cavidad peritoneal 2,5 $\pm 0,31$ de Voluven $^{\circledR}$ para obtener la PIA objetivo al final de la fase experimental $(p<0,001)$. Paralelamente hubo una disminución de la PPA de $72,3 \pm 3,2$ a $57,3 \pm 4,0 \mathrm{mmHg}(\mathrm{p}<0,001)$.

\section{Monitorización hemodinámica}

La inducción del SCA no indujo cambios significativos en la FC, la PAM ni en la PVC. Hubo una disminución significativa del GC de $5,19 \pm 0,33$ a $4,34 \pm 0,281 / \mathrm{min} / \mathrm{m}^{2}(\mathrm{p}=0,01)$, sin alteraciones significativas en el GEDV, VSI e IRSV (tabla 1). La $\mathrm{DO}_{2}$ disminuyó de $615 \pm 109$ a $505 \pm 94 \mathrm{ml} / \mathrm{min} / \mathrm{m}^{2}(\mathrm{p}=0,008)$.

\section{Monitorización respiratoria}

Posterior a la inducción de SCA hubo una disminución significativa en la $\mathrm{PaO}_{2}(421 \pm 10$ a $369 \pm 28 \mathrm{mmHg}, \mathrm{p}=0,008)$, sin cambios en la $\mathrm{PaCO}_{2}$. Concomitantemente hubo un aumento significativo de la presión diferencial $(9,7 \pm$ 0,6 a $\left.19,9 \pm 1,3 \mathrm{cmH}_{2} \mathrm{O}, \mathrm{p}=0,002\right)$, la presión plateau $\left(14,7 \pm 0,6\right.$ a 24,9 $\pm 1,3 \mathrm{cmH}_{2} \mathrm{O}$, $\mathrm{p}=0,002)$ y la PMVA $(7,1 \pm 0,2$ a $8,8 \pm 0,2$ $\left.\mathrm{cmH}_{2} \mathrm{O}, \mathrm{p}=0,002\right)$. La compliance estática se redujo de $1,28 \pm 0,09$ a $0,62 \pm 0,04 \mathrm{ml} / \mathrm{cmH}_{2} \mathrm{O} /$ $\mathrm{kg}(\mathrm{p}=0,002)$ y el IO aumentó de 1,69 $\pm 0,06$ a $5,47 \pm 1,03 \mathrm{cmH}_{2} \mathrm{O} / \mathrm{mmHg}(\mathrm{p}=0,002)($ tabla 2).

\section{Tabla 1. Consecuencias hemodinámicas de síndrome compartimental abdominal en modelo experimental pediátrico}

\begin{tabular}{|lccc|}
\hline & Basal & SCA & p-value \\
\hline FC (latidos por min) & $134 \pm 5$ & $123 \pm 5$ & 0,52 \\
PAM (mmHg) & $77 \pm 3$ & $82 \pm 4$ & 0,146 \\
PVC (mmHg) & $11,1 \pm 0,6$ & $11,7 \pm 0,9$ & 0,809 \\
PPA (mmHg) & $72,3 \pm 3,2$ & $57,3 \pm 4,0$ & $<0,001$ \\
GC (I/min/m $\left.{ }^{2}\right)$ & $5,19 \pm 0,33$ & $4,34 \pm 0,28$ & 0,01 \\
GEDV (ml/m²) & $391 \pm 20$ & $367 \pm 31$ & 0,99 \\
VSI (ml/m²) & $40,5 \pm 1,86$ & $38,3 \pm 2,4$ & 0,345 \\
IRSV (dinas.seg.cm $\left.{ }^{2}\right)$ & $1032 \pm 106$ & $1276 \pm 120$ & 0,12 \\
\hline
\end{tabular}

SCA: síndrome compartimental abdominal, FC: frecuencia cardíaca, PAM: presión arterial media, PVC: presión venosa central, GC: gasto cardiaco indexado, GEDV: volumen global diastólico final, VSI: volumen sistólico indexado, IRSV: resistencia vascular sistémica indexada. Resultados expresados como media \pm SEM. Consideramos significativo un $p<0,05$.

\section{Tabla 2. Consecuencias respiratorias de síndrome compartimental abdominal en modelo experimental pediátrico}

\begin{tabular}{|lccc|}
\hline & Basal & SCA & p-value \\
$\mathrm{PaO}_{2}(\mathrm{mmHg})$ & $421 \pm 10$ & $369 \pm 28$ & 0,008 \\
$\mathrm{PaCO}_{2}(\mathrm{mmHg})$ & $42 \pm 2$ & $44 \pm 2$ & 0,158 \\
$\mathrm{IO}$ & $1,69 \pm 0,06$ & $5,47 \pm 1,03$ & 0,002 \\
$\mathrm{PD}\left(\mathrm{cmH}_{2} \mathrm{O}\right)$ & $9,7 \pm 0,6$ & $19,9 \pm 1,3$ & 0,002 \\
$\mathrm{P}$ & $14,7 \pm 0,6$ & $24,9 \pm 1,3$ & 0,002 \\
$\mathrm{PM}\left(\mathrm{cmH}_{2} \mathrm{O}\right)$ & $7,1 \pm 0,2$ & $8,8 \pm 0,2$ & 0,002 \\
\hline $\mathrm{C}_{\mathrm{RS}}\left(\mathrm{ml} / \mathrm{cmH}_{2} \mathrm{O} / \mathrm{kg}\right)$ & $1,28 \pm 0,09$ & $0,62 \pm 0,04$ & 0,002 \\
\hline
\end{tabular}

SCA: síndrome compartimental abdominal, $\mathrm{PaO}_{2}$ : presión arterial de oxígeno, $\mathrm{PaCO}_{2}$ : presión arterial de dióxido de carbono, IO: índice de oxigenación, PD: presión diferencial, $\mathrm{P}_{\mathrm{PL}}$ : presión plateau, PMVA: presión media en la vía aérea, $C_{R s}$ : compliance del sistema respiratorio. Resultados expresados como media \pm SEM. Consideramos significativo un $p<0,05$.

\section{Discusión}

En este modelo experimental pediátrico euvolémico de síndrome compartimental abdominal secundario a una súbita ocupación líquida de la cavidad peritoneal, los principales hallazgos fueron los siguientes: 1) ocurrió una reducción del GC de un $16 \%$ y una disminución de la $\mathrm{DO}_{2}$ de un $18 \%$ no detectados por la monitorización convencional, y 2) hubo un deterioro de la mecánica pulmonar, caracterizado por una reducción cercana al $50 \%$ de la 
compliance del sistema respiratorio asociado a un incremento significativo en las presiones plateau, diferencial y media de la vía aérea, influyendo en una disminución de la $\mathrm{PaO}_{2}$ e incremento del índice de oxigenación.

Respecto a los efectos hemodinámicos, nuestros resultados concuerdan con lo reportado en la literatura. Está descrito que el aumento de la PIA produce una desviación cefálica del diafragma, aumentando por contigüidad la presión intratorácica y disminuyendo secuencialmente el retorno venoso y el gasto cardíaco, fenómeno más acentuado en sujetos hipovolémicos. Tales reducciones están descritas con PIA mayores o iguales a $10 \mathrm{mmHg}^{19}$. Como se ha evidenciado en pacientes pediátricos, la reducción del flujo venoso de la vena cava inferior, secundario a compresión directa de las venas suprahepáticas y porta, también puede contribuir en este fenómeno ${ }^{20}$. Adicionalmente, el aumento de la presión intratorácica se transmite hacia la fosa pericárdica y comprime al corazón reduciendo la presión transmural ventricular así como el volumen telediastólico biventricular (precarga). Paradójicamente esta reducción de la precarga no se refleja en una disminución de la PVC, como ocurrió en nuestro modelo, dificultando una adecuada estimación de la primera y postulándose que serían más sensibles los métodos volumétricos que los manométricos para estimar el volumen intravascular ${ }^{21-23}$. En nuestro modelo no se logró identificar cambios en los marcadores volumétricos de precarga (GEDV y VSI), aunque debemos destacar que la condición de "precarga optimizada" pudo haber minimizado este efecto.

La elevación de la PIA aumenta la resistencia vascular sistémica (RVS) y pulmonar (RVP) debido a compresión directa de las arteriolas intra-abdominales y parénquima pulmonar respectivamente (postcarga). En nuestro modelo el incremento de la RVS no fue significativo, probablemente atribuido a la corta duración del modelo y/o a un tamaño de la muestra insuficiente. A pesar de la reducción del retorno venoso y gasto cardíaco, algunos pacientes pueden mantener una PAM estable frente a elevaciones moderadas de la PIA, como ocurrió en este modelo. Sin embargo, aquellos pacientes con una reserva cardiovascular reducida o límite pueden ser incapaces de compensar grandes aumentos de la postcar$\mathrm{ga}^{21}$. De este modo, en sujetos con función cardiovascular conservada, la hipotensión es una manifestación tardía de SCA. Estas observaciones son muy relevantes si consideramos que los signos clínicos para reconocer una entrega de oxígeno inadecuada son inespecíficos y la capacidad para estimar un GC reducido en niños sólo con examen físico es muy limitada ${ }^{24}$. Basado en que la mayor parte de los órganos son capaces de mantener un flujo sanguíneo relativamente constante en un rango amplio de presión, Cheatham propone que la presión de perfusión abdominal sería una mejor meta para guiar la resucitación en pacientes con $\mathrm{SCA}^{25}$. Concordantemente en nuestro estudio la PPA disminuyó un $20 \%$ tras la inducción del SCA.

Respecto a los efectos respiratorios, se evidenció un deterioro de la mecánica pulmonar asociado a un empeoramiento incipiente del intercambio gaseoso. El aumento de la PIA eleva al diafragma en forma pasiva, transmitiéndose dicha presión hacia los lóbulos pulmonares inferiores, modificando la mecánica tóraco-pulmonar. El efecto restrictivo de este fenómeno provoca una disminución de la distensibilidad pulmonar y capacidad residual funcional (CRF), promueve la discordancia entre ventilación y perfusión e incrementa las presiones de la vía aérea, como ocurrió en este modelo experimental. Estas alteraciones ocurren con PIA iguales o mayores a $15 \mathrm{mmHg}^{26}$. En niños esto sería responsable de hipoxemia y en ocasiones de acidosis respiratoria, de manera similar a lo descrito en adultos ${ }^{27}$. Es importante destacar la discrepancia entre la magnitud de los efectos sobre mecánica pulmonar e intercambio gaseoso, los cuales explicamos por la breve duración de este modelo. Adicionalmente, las alteraciones graves del intercambio gaseoso previamente descritas no serían una manifestación temprana de un SCA.

Las consecuencias clínicas pueden ser muy relevantes. La hipertensión intra-abdominal incrementa las presiones pleurales e intratorácica, generando edema y atelectasias. De este modo se reduce la CRF, asemejando una enfermedad pulmonar restrictiva. En consecuen- 
cia tenemos hipoxemia, hipercapnia, shunt intrapulmonar e incremento de la ventilación de espacio muerto, siendo estas alteraciones reversibles con ajuste adecuado de parámetros de ventilación mecánica, destinado a restablecer la CRF mientras se resuelve la causa subyacente $^{11,27}$.

Debemos señalar que las principales limitaciones de este estudio fueron las siguientes: a) Dado que este es un modelo de corta duración, la magnitud de las disfunciones orgánicas probablemente subestiman las repercusiones posibles en niños críticamente enfermos, donde la hipertensión intra-abdominal (HIA) persiste por muchas horas o días; sin embargo, estos cambios respiratorios y circulatorios fueron similares a los descritos por HIA durante cirugía laparoscópica ${ }^{28}$; b) La ocupación de la cavidad peritoneal por un fluido de escaso efecto biológico local pretende simular un SCA en contexto de patologías "médicas", siendo probablemente mayor la repercusión hemodinámica ante ocupación piógena o hemática; c) Consideramos sólo las consecuencias hemodinámicas bajo una condición previa de euvolemia y función cardiovascular normal, siendo conocido que los efectos de un SCA son más acentuados en hipovolemia y/o disfunción miocárdica, alteraciones frecuentes en niños graves; d) El empleo de TDTP en lugar de termodilución pulmonar (catéter de arteria pulmonar) para monitorización hemodinámica avanzada, el primero está suficientemente validado en pacientes y modelos pediátricos en la actualidad $^{29-31}$, además al no emplear catéter de arteria pulmonar, tampoco pudimos medir resistencia vascular pulmonar, la que aumenta en adultos con $\mathrm{SCA}^{21}$; e) No se midió la presión pleural dada las dificultades técnicas en la instalación y transducción de catéteres esofágicos de tamaño pediátrico. Esto nos hubiera permitido discriminar los efectos del SCA sobre la compliance de la pared torácica y pulmonar; f) No medimos marcadores de perfusión regional, alteración relevante en SCA; futuros estudios debieran cuantificar su impacto. Finalmente, debemos agregar las limitaciones propias de extrapolar la información desde modelos experimentales a pacientes gravemente enfermos.

A pesar de esto nos parece de gran impor- tancia monitorizar la PIA en pacientes con factores de riesgo para desarrollar un SCA (cirugía abdominal mayor, trauma abdominal, grandes quemados, enterocolitis necrotizante, shock refractario, etc), más aún frente al desarrollo o progresión de la disfunción hemodinámica y/o respiratoria, aunque estas no sean graves. Concordantemente es fundamental la monitorización seriada de funciones orgánicas posterior al desarrollo de un incremento súbito en la presión intra-abdominal. Esto es fundamental si consideramos que las disfunciones orgánicas desencadenadas por un SCA son potencialmente reversibles en niños y una intervención insuficiente o tardía está asociada a una mayor mortalidad $3,5,32,33$.

\section{Referencias}

1.- Beck R, Halberthal M, Zonis Z, Shoshani G, Hayari L, Bar-Joseph G: Abdominal compartment syndrome in children. Pediatr Crit Care Med 2001; 2: 51-6.

2.- Ejike JC, Humbert S, Bahjri K, Mathur M: Outcomes of children with abdominal compartment syndrome. Acta Clin Belg Suppl 2007; 1: 141-8.

3.- Pearson EG, Rollins MD, Vogler $S A$, et al: Decompressive laparotomy for abdominal compartment syndrome in children: before it is too late. J Pediatr Surg 2010; 45: 1324-9.

4.- Malbrain ML, Cheatham ML, Kirkpatrick A, et al: Results from the International Conference of Experts on Intra-abdominal Hypertension and Abdominal Compartment Syndrome. I. Definitions. Intensive Care Med 2006; 32: 1722-32.

5.- Carlotti AP, Carvalho WB: Abdominal compartment syndrome: A review. Pediatr Crit Care Med 2009; 10: 115-20.

6.- Akhobadze GR, Chkhaidze MG, Kanjaradze DV, Tsirkvadze IB, Ukleba VA: Identification, management and complications of intra-abdominal hypertension and abdominal compartment syndrome in neonatal intensive care unit (a single center retrospective analysis). Georgian Med News 2011; 192: 58-64.

7.- Ejike JC, Newcombe J, Baerg J, Bahjri K, Mathur M: Understanding of Abdominal Compartment Syndrome among Pediatric Healthcare Providers. Crit Care Res Pract 2010: 1-6.

8.- Malbrain ML Chiumello D, Pelosi P, et al: Prevalence of intra-abdominal hypertension in critically ill patients: 
a multicenter epidemiological study. Intensive Care Med 2004; 30: 822-9.

9.- Balogh Z, McKinley BA, Holcomb JB, et al: Both primary and secondary abdominal compartment syndrome can be predicted early and are harbingers of multiple organ failure. J Trauma 2003; 54: 848-59.

10.- Balogh Z, McKinley BA, Cocanour CS, et al: Secondary abdominal compartment syndrome is an elusive early complication of traumatic shock resuscitation. Am J Surg 2002; 184: 538-43.

11.- Tomicic V, Cruces P, Donoso A: Síndrome compartimental del abdomen en el paciente crítico. Rev Chil Pediatr 2006; 77: 557-67.

12.- Hedenstierna $G$, Larsson A: Influence of abdominal pressure on respiratory and abdominal organ function. Curr Opin Crit Care 2012; 18: 80-5.

13.- Cheatham ML: Abdominal compartment syndrome: pathophysiology and definitions. Scand J Trauma Resusc Emerg Med 2009; 2;17:10.

14.- Lui F, Sangosanya A, Kaplan LJ: Abdominal compartment syndrome: clinical aspects and monitoring. Crit Care Clin 2007; 23: 415-33.

15.- Renner J, Meybohm P, Gruenewald M, et al: Global end-diastolic volume during different loading conditions in a pediatric animal model. Anesth Analg 2007; 105: 1243-9.

16.- López-Herce J, Ruperez M, Sánchez C, García C, Garcia $E$ : Estimation of the parameters of cardiac function and of blood volume by arterial thermodilution in an infant animal model. Paediatr Anaesth 2006; 16: 63540.

17.- Kron IL, Harman PK, Nolan SP: The measurement of intra-abdominal pressure as a criterion for abdominal re-exploration. Ann Surg 1984; 199: 28-30.

18.- Roch A, Michelet $P$, Lambert D, et al: Accuracy of the double indicator method for measurement of extravascular lung water depends on the type of acute lung injury. Crit Care Med 2004; 32: 811-7.

19.- Ivatury RR, Diebel LN, Porter JM, et al: Intra-abdominal hypertension and the abdominal compartment syndrome. Surgical Clin North Am 1997; 77: 783-800.

20.- Epelman M, Soudack M, Engel A, Halberthal M, Beck $R$ : Abdominal compartment syndrome in children: CT findings. Pediatr Radiol 2002; 32: 319-22.

21.- Malbrain ML, Coninck J, Debaveye Y, et al: Optimal preload markers in intra-abdominal hypertension. In- tensive Care Med 2001; 27: S202.

22.- Schachtrupp A, Graf J, Tons C, et al: Intravascular volume depletion in a 24 -hour porcine model of intraabdominal hypertension. J Trauma 2003; 55:734-40.

23.- Malbrain $M L$, Nieuwendijk R, Verbrugghe $W$, et al: Effect of intra-abdominal pressure on pleural and filling pressure. Intensive Care Med 2003; 29: S73.

24.- Tibby SM, Hatherill M, Marsh MJ, Murdoch IA: Clinicians' abilities to estimate cardiac index in ventilated children and infants. Arch Dis Child 1997; 77: 516-8.

25.- Cheatham ML, White MW, Sagraves SG, Johnson JL, Block EF: Abdominal perfusion pressure: a superior parameter in the assessment of intra-abdominal hypertension. J Trauma 1999; 49: 621-7.

26.- Mutoh T, Lamm WJ, Embree LJ, Hildebrandt J, Albert $P K$ : Volume infusion produces abdominal distension, lung compression, and chest wall stiffening in pigs. J Appl Physiol 1992; 72: 575-82.

27.- Díaz FJ, Fernández Sein A, Gotay F: Identification and management of Abdominal Compartment Syndrome in the Pediatric Intensive Care Unit. P R Health Sci J 2006; 25: 17-22.

28.- Baroncini S, Gentili A, Pigna A, et al: Anaesthesia for laparoscopic surgery in paediatrics. Minerva Anestesiol 2002; 68: 406-13.

29.- Tibby SM, Hatherill M, Marsh MJ, Morrison G, Anderson D, Murdoch IA: Clinical validation of cardiac output measurements using femoral artery thermodilution with direct Fick in ventilated children and infants. Intensive Care Med 1997; 23: 987-91.

30.- Rupérez M, López-Herce J, García C, Sánchez C, García E, Vigil D: Comparison between cardiac output measured by the pulmonary arterial thermodilution technique and that measured by the femoral arterial thermodilution technique in a pediatric animal model. Pediatr Cardiol 2004; 25: 119-23.

31.- Lemson J, de Boode WP, Hopman JC, Singh SK, van der Hoeven $J G$ : Validation of transpulmonary thermodilution cardiac output measurement in a pediatric animal model. Pediatr Crit Care Med 2008; 9: 313-9.

32.- DeCou JM, Abrams RS, Miller RS, Gauderer MW: Abdominal compartment syndrome in children: experience with three cases. J Pediatr Surg 2000; 35: 840-2.

33.- Steinau G, Kaussen T, Bolten B, et al: Abdominal compartment syndrome in childhood: diagnostics, therapy and survival rate. Pediatr Surg Int 2011; 27: 399-405. 